\title{
Hepatic and Splenic Volumetry Could Be Used as an Imaging Parameter to Evaluate Fibrosis Grades of the Diffuse Liver Disease Including Nonalcoholic Fatty Liver Disease
}

\author{
Woo Kyoung Jeong \\ Department of Radiology and Center for Imaging Science, Samsung Medical Center, Sungkyunkwan University School of Medicine, Seoul, \\ Korea \\ See "Noninvasive Assessment of Advanced Fibrosis Based on Hepatic Volume in Patients with Nonalcoholic Fatty Liver Dis- \\ ease" by Tatsuya Hayashi, et al. on page 674, Vol. 11. No. 5, 2017
}

Morphologic change of the liver is well known in the patients who underwent chronic liver disease. ${ }^{1}$ The subjective finding of liver cirrhosis is hypotrophy of right liver accompanying with hypertrophy of left liver and caudate lobe. On the cross-sectional images, the right liver exhibited relatively greater shrinkage, while the caudate lobe underwent relative enlargement. Some researchers speculate that one cause of hypertrophy of the caudate lobe and atrophy of the right is thought to be changes in their blood supply. Although most portal branches distributing to the caudate lobe arise from the left portal branch or from the bifurcation of the portal vein, there are branches from the right main portal vein and even the posterior segmental branch. The right portal venous bifurcation might more accurately divide the hypertrophied caudate and central liver from the atrophied right lobe than does the main portal venous bifurcation. ${ }^{2}$

In the article included in this volume, Hayashi et al. ${ }^{3}$ addressed the volumetric left lobe to right lobe (L/R) ratio in patients with nonalcoholic fatty liver disease demonstrated better performance in differentiating cirrhosis compared with other serologic tests such as aspartate aminotransferase to platelet ratio index (APRI) and BARD scores. The most anticipated aspect of this study is volumetric ratio is more reliable to obtain the parameters from morphologic features because it is not measure at specific cross-section; and automatic segmentation by computerized algorithm is more reliable than human work. Moreover, interobserver and intraobserver variability issue could be overcome by computerized volumetric measurement. In neurologic or neuropsychiatric researches, magnetic resonance (MR)-based volumetric parameters using brain magnetic resonance imaging (MRI) have been developed and widely used for diagnosis and prediction of clinical outcomes. ${ }^{4}$

Hepatic volumetry using three-dimensional image data is a method to quantify the morphologic change of the liver. The use of more objective methods as computed tomography (CT) or MRI seems fundamental. These two imaging techniques showed a very good accuracy in the estimation of the graft dimensions before transplant. Accurate segmentation of the liver is crucial, but it is not easy to manually draw following the boundary of the liver. There are several approaches to achieved reasonably segmentation automatically or semiautomatically in recent years, such as using statistical model, textual information, and atlas of standardized liver model. The volumetric values obtained with automated or semiautomated method agreed with those obtained with manual method, and lap time for automated volumetry substantially reduced compared with manual method. $^{5}$

However, the result of automatic segmentation of the abdominal organs can often be overestimated because the contrast enhancement of the liver is similar to that of the adjacent organs such as spleen, abdominal wall muscle, and the stomach and they can be included in the result of liver volumetry. Recently, gadoxetic acid (Gd-EOB-DTPA) enhanced MRI enables to easily segment the liver volume because it makes the only liver parenchyma appear uniformly bright on T1-weighted image. Lee et al. ${ }^{6}$ addressed the average volume measurement error of this method was 2.35\% and total processing time was about 12

Correspondence to: Woo Kyoung Jeong

Department of Radiology and Center for Imaging Science, Samsung Medical Center, Sungkyunkwan University School of Medicine, 81 Irwon-ro, Gangnam-gu, Seoul 06351, Korea

Tel: +82-2-3410-1923, Fax: +82-2-3410-0049, E-mail: jeongwk@gmail.com pISSN 1976-2283 eISSN 2005-1212 https://doi.org/10.5009/gnl17333

(a) This is an Open Access article distributed under the terms of the Creative Commons Attribution Non-Commercial License (http://creativecommons.org/licenses/by-nc/4.0) which permits unrestricted non-commercial use, distribution, and reproduction in any medium, provided the original work is properly cited. 
seconds.

There are several papers to evaluate the relationship between hepatic volume and liver fibrosis. Right liver and left medial segment volumes tended to decrease, and the ratio of caudate to total liver volume tended to increase, ${ }^{7}$ and the ratio of Couinaud segment I-III to segment IV-VIII increased with stage of fibrosis. ${ }^{8}$ Spleen volume is also important values to estimate the fibrosis stages. Splenomegaly generally reflects the presence of portal hypertension, but it is not always correlated with the portal hypertension grades because the combination of splenic congestion and tissue hyperplasia affect the splenic volume. Therefore, some researchers understand the combination of hepatic and splenic volume can be a parameter for estimation of the severity of liver cirrhosis, and there are some reports which addressed that splenic volume and the ratio of splenic to liver volume could estimate fibrosis stages ${ }^{7,8}$ and the severity of portal hypertension. ${ }^{9}$ The measurement of splenic volume is also not easy because of the same reason of the liver, but Lee et al. ${ }^{10}$ introduced a useful method to measure the splenic volume using diffusion-weighted MR imaging.

However, as appears by Hayashi's paper, there are still remained some problems of volumetric evaluation of diffuse liver diseases. First of all, it may be a time-consuming and unreproducible job unless a robust automatic segmentation method is perfectly realized. Second, volumetric evaluation of hepatic fibrosis is not applicable to the patients with non-cirrhotic liver fibrosis (F1 to F3) because the change of hepatic volume develops in advanced fibrosis stage. MR or ultrasound elastography can play a supplementary role for the discrimination of the lowgrade fibrosis.

In conclusion, it is very encouraging that parametric imaging including hepatic and splenic volumetry is increasing to evaluate the severity of diffuse liver diseases such as hepatic fibrosis and portal hypertension. However, it should be easily applicable and reliable, and the reference value of the parametric imaging should be generally acceptable. I hope that various imaging parameters for help to find clinically significant result would be developed on the chronic liver disease.

\section{CONFLICTS OF INTEREST}

No potential conflict of interest relevant to this article was reported.

\section{REFERENCES}

1. Harbin WP, Robert NJ, Ferrucci JT Jr. Diagnosis of cirrhosis based on regional changes in hepatic morphology: a radiological and pathological analysis. Radiology 1980;135:273-283.

2. Awaya H, Mitchell DG, Kamishima T, Holland G, Ito K, Matsumoto T. Cirrhosis: modified caudate-right lobe ratio. Radiology 2002;224:769-774.

3. Hayashi T, Saitoh S, Fukuzawa K, et al. Noninvasive assessment of advanced fibrosis based on hepatic volume in patients with nonalcoholic fatty liver disease. Gut Liver 2017;11:674-683.

4. Greven CU, Bralten J, Mennes M, et al. Developmentally stable whole-brain volume reductions and developmentally sensitive caudate and putamen volume alterations in those with attentiondeficit/hyperactivity disorder and their unaffected siblings. JAMA Psychiatry 2015;72:490-499.

5. Suzuki K, Epstein ML, Kohlbrenner R, et al. Quantitative radiology: automated CT liver volumetry compared with interactive volumetry and manual volumetry. AJR Am J Roentgenol 2011; 197:W706-W712.

6. Lee J, Kim KW, Kim SY, et al. Feasibility of semiautomated MR volumetry using gadoxetic acid-enhanced MRI at hepatobiliary phase for living liver donors. Magn Reson Med 2014;72:640-645.

7. Li WX, Zhao XT, Chai WM, et al. Hepatitis B virus-induced liver fibrosis and cirrhosis: the value of liver and spleen volumetry with multi-detector spiral computed tomography. J Dig Dis 2010;11: 215-223.

8. Pickhardt PJ, Malecki K, Hunt OF, et al. Hepatosplenic volumetric assessment at MDCT for staging liver fibrosis. Eur Radiol 2017;27:3060-3068.

9. Iranmanesh P, Vazquez 0, Terraz S, et al. Accurate computed tomography-based portal pressure assessment in patients with hepatocellular carcinoma. J Hepatol 2014;60:969-974.

10. Lee J, Kim KW, Lee H, et al. Semiautomated spleen volumetry with diffusion-weighted MR imaging. Magn Reson Med 2012;68:305310 